\title{
Genes and Their Effects on Dental Caries May Differ between Primary and Permanent Dentitions
}

\author{
X. Wang ${ }^{a, b, h}$ J.R. Shaffer ${ }^{d}$ R.J. Weyant ${ }^{c, h} \quad$ K.T. Cuenco ${ }^{a, b, d, h} \quad$ R.S. DeSensi ${ }^{a, b, h}$ \\ R. Crout ${ }^{f, h}$ D.W. McNeilg M.L. Marazita ${ }^{a, b}, d, e, h$ \\ ${ }^{a}$ Center for Craniofacial and Dental Genetics, ${ }^{b}$ Department of Oral Biology, 'Department of Dental Public Health \\ and Information Management, School of Dental Medicine, ${ }^{d}$ Department of Human Genetics, Graduate School of \\ Public Health, and ${ }^{\mathrm{e}} \mathrm{Clinical}$ and Translational Science Institute and Department of Psychiatry, School of Medicine, \\ University of Pittsburgh, Pittsburgh, Pa.; ${ }^{f}$ Department of Periodontics, School of Dentistry, and ${ }^{9}$ Dental Practice \\ and Rural Health, West Virginia University, Morgantown, W. Va.; ${ }^{\text {h} C e n t e r ~ f o r ~ O r a l ~ H e a l t h ~ R e s e a r c h ~ i n ~ A p p a l a c h i a, ~}$ \\ University of Pittsburgh, Pittsburgh, Pa., and West Virginia University, Morgantown, W. Va., USA
}

\section{Key Words}

Dental caries · Genetics · Heritability · Permanent

dentition - Primary dentition

\begin{abstract}
The importance of genetic factors in the genesis of dental caries of both primary and permanent dentitions is well established; however, the degree to which genes contribute to the development of dental caries, and whether these genes differ between primary and permanent dentitions, is largely unknown. Using family-based likelihood methods, we assessed the heritability of caries-related phenotypes for both children and adults in 2,600 participants from 740 families. We found that caries phenotypes in the primary dentition were highly heritable, with genes accounting for $54-70 \%$ of variation in caries scores. The heritability of caries scores in the permanent dentition was also substantial (35-55\%, all $p<0.01$ ), although this was lower than analogous phenotypes in the primary dentition. Assessment of the genetic
\end{abstract}

\section{KARGER}

() 2010 S. Karger AG, Basel

Fax +41613061234 E-Mail karger@karger.ch www.karger.com www.karger.com/cre correlation between primary and permanent caries scores indicated that $18 \%$ of the covariation in these traits was due to common genetic factors $(p<0.01)$. Therefore, dental caries in primary and permanent teeth may be partly attributable to different suites of genes or genes with differential effects. Sex and age explained much of the phenotypic variation in permanent, but not primary, dentition. Further, including pre-cavitated white-spot lesions in the phenotype definition substantially increased the heritability estimates for dental caries. In conclusion, our results show that dental caries are heritable, and suggest that genes affecting susceptibility to caries in the primary dentition may differ from those in permanent teeth. Moreover, metrics for quantifying caries that incorporate white-spot lesions may serve as better phenotypes in genetic studies of the causes of tooth decay.

Copyright $\odot 2010$ S. Karger AG, Basel

Co-first authors X.W. and J.R.S. contributed equally to this work.
Mary L. Marazita, PhD

Center for Craniofacial and Dental Genetics, University of Pittsburgh

Suite 500 Bridgeside Point, 100 Technology Dr.

Pittsburgh, PA 15219 (USA)

Tel. +1 412648 8380, Fax +1 412648 8779, E-Mail marazita@ pitt.edu 
The etiology of dental caries is thought to involve a complex interplay of environmental (including behavioral) and genetic factors. Although the environmental risk factors for dental caries have been studied for many years [Hunter, 1988; Anderson, 2002], the role of genes in the development of dental caries is largely unknown.

Pioneer twin studies investigating the heritability (i.e. proportion of variation due to genes) of dental caries in both children [Liu et al., 1998; Bretz et al., 2005a, 2005b, 2006] and adults [Horowitz et al., 1958; Goodman et al., 1959; Boraas et al., 1988; Shuler, 2001] have clearly supported the key role of genetics in tooth decay. However, the robustness of these studies was limited due to their small sample sizes and their exclusive use of twins (which can lead to inflated heritability estimates). Moreover, because previous studies focused largely on either children or adults, a comprehensive comparison of the influence of genetic factors on dental caries in primary versus permanent dentition has not been performed. It is unclear whether the heritability of tooth decay is similar for primary and permanent dentitions, and whether the same or different genes are involved.

Moreover, dental caries is an especially complex phenotype that cannot be readily summarized into a single, biologically meaningful, metric. Is it currently unknown which methods of quantifying dental caries best reflect the underlying genetic risk and would be most appropriate for future investigations of the specific genes influencing tooth decay.

To address these questions, we performed a comprehensive heritability analysis of caries-related phenotypes for both primary and permanent dentitions in 2,600 participants from 740 families. We explored multiple metrics for quantifying dental caries. The data used for this project were drawn from the ongoing oral health etiology studies of the Center for Oral Health Research in Appalachia (COHRA), a partnership between the University of Pittsburgh and West Virginia University.

\section{Subjects and Methods}

\section{Recruitment}

The Center for Oral Health Research in Appalachia (COHRA) was initiated to study the contributions of individual, family, and community factors to oral diseases in the Appalachian population. Details regarding this sample have been previously reported [Polk et al., 2008]. COHRA participants were recruited from two central West Virginia counties (Webster and Nicholas) and three western Pennsylvania counties (Allegheny, Washington and McKean). Eligible households had at least one parent-child pair in which the participating child was the biological offspring of a par- ticipating parent and was between the ages of 1 and 18 years. Participants were recruited without regard to their oral health status, and all members of eligible households were accepted into the study regardless of biological and/or legal relationships. Exclusion criteria included individuals with neurological impairment, severe physical or intellectual handicap, or psychosis, and families where one member of the biological parent-offspring pair had reduced capacity to resist infection or form blood clots. Written informed consent (or assent from children, with parental consent) was obtained from participants using forms and procedures approved by the COHRA research committee as well as the University of Pittsburgh and West Virginia University institutional review boards.

A total of 732 households, which included 2,600 individuals from 740 distinct biological families, were enrolled. Family sizes ranged from 1 to 20 individuals, with mean family size of 4.72 members. In total, 3,232 relative pairs were analyzed, including 1,817 parent-offspring pairs, 756 siblings, 347 half-siblings, 120 avuncular pairs, 104 first cousins, and 88 other relative pairs.

In general, the Appalachian population ranks very low compared to the nation as a whole in terms of many oral heath measures and access to oral health services [Grembowski et al., 1987; Huttlinger and Purnell, 1998; Janes et al., 1999; Centers for Disease Control and Prevention 1999, 2003]. While our sample is fairly representative of the Appalachian population, including households from a range of socioeconomic statuses, the majority of families are low-income (median annual household income less than USD 25,000). Note, however, that we also believe the Northern Appalachian region differs from many other areas of the USA only in the degree to which these stressors are concentrated in the population; thus, the results from our research may be broadly generalizable.

To validate the reported familial relationships in our sample, we analyzed 72 ancestry-informative single nucleotide polymorphisms genotyped by the Center for Inherited Disease Research at Johns Hopkins University [Marosy et al., 2007]. Using standard relationship-testing methods [O'Connell and Weeks, 1998] (not described herein) we identified and corrected family relationship errors before performing the analyses reported here.

\section{Data Collection}

All dental exams were conducted by dentists or research dental hygienists who were calibrated at least annually to one of the dentists who served as the calibration reference. Mean inter- and intra-rater concordance of caries assessments were high [Polk et al., 2008]. After drying of teeth (gauze or air) if needed, each surface of each tooth was scored by visual inspection using a dental mirror and artificial light. No explorer was used for occlusal or buc$\mathrm{cal} /$ lingual surfaces. If interproximal caries were suspected, then an explorer was used if necessary. No radiographs were taken.

As per the World Health Organization DMFT/dft scale, each surface was scored as pre-cavitated (white spot) or decayed (according to the four-level classification system), filled, missing due to decay, hypoplastic, or missing due to reasons other than decay. To distinguish white spots from hypoplasia, the location and the characteristics of the lesion were considered. For example, characteristics of white spots include chalking, softening, or breaking in the enamel surface, none of which are characteristics of hypoplasia. Moreover, white spots commonly occur in areas prone to decay (e.g. pits and fissures), whereas hypoplasia more 
often occurs in areas which are prone to trauma during development (e.g. facial surface of an anterior tooth) and may have a group of small pits. From this assessment, the following two composite 'caries scores' were derived: (1) permanent DMFT score, which is a summation of D, M, and F permanent teeth, and (2) permanent proportion DMFT score, which was calculated as the DMFT score divided by the total number of permanent teeth for which we have data (i.e. teeth present plus teeth missing due to decay). Two composite variables were similarly calculated for primary dentition with the exception that the m component was not included in primary $\mathrm{dft}$ and primary proportion $\mathrm{dft}$ scores due to possible misidentification of un-erupted primary teeth. Likewise, the composite variables were calculated for 'total' dentition $(\mathrm{DMFT}+\mathrm{dft}$, etc.), which included both permanent and primary dentition together for those participants in the mixed dentition (i.e. having both permanent and primary teeth present). Finally, two forms of caries scores were calculated which either included (D1MFT/d1ft) or excluded (D2MFT/d2ft) white-spot lesions, which are considered pre-cavitation lesions. Permanent and primary caries scores were analyzed for individuals based on the presence of teeth of each dentition type, regardless of age. Individuals who did not have any teeth present were excluded from these analyses.

\section{Statistical Analysis}

Heritability analyses were performed within a variance components framework, which partitions the phenotypic variance into environmental, heritable and residual error components while conditioning on the pedigree structure of the data. The general form of this model is:

$$
\mathrm{y}_{\mathrm{i}}=\mu+\sum_{j}^{n} \beta_{\mathrm{j}} \mathrm{X}_{\mathrm{ji}}+\mathrm{g}_{\mathrm{i}}+\mathrm{e}_{\mathrm{i}},
$$

where $y_{i}$ is the dental phenotype for the $i^{\text {th }}$ individual, $\mu$ is the sample mean, $X_{j i}$ are the values of the predictors, $j$, for the $i^{\text {th }}$ individual, $\beta_{j}$ are the regression coefficients for the $\mathrm{j}^{\mathrm{th}}$ predictor, $\mathrm{g}_{\mathrm{i}}$ is the individual additive polygenic effect (based on expected genetic sharing due to the familial relationship between relative pairs), and $e_{i}$ is the individual residual error effect [Almasy and Blangero, 1998]. Model parameters were estimated using pedigree-based likelihood methods, from which residual heritability $\left(\mathrm{h}^{2}\right.$; the proportion of phenotype variance due to genetic factors after adjustment for age and sex) was calculated. The significance of the sex parameter (indicating a difference between males and females) was assessed via the likelihood ratio test, which compares the full model (where the sex parameter is estimated) to the constrained model (i.e. where the sex parameter is set to zero) and follows the $\chi^{2}$ distribution with 1 degree of freedom.

The significance of heritability was assessed empirically via analysis of 100 phenotype permutations to avoid inflation of the theoretical asymptotic $p$ values that could result from deviations of the phenotype distributions from normality.

A bivariate extension of the univariate model above was used to estimate an additional parameter, genetic correlation $\left(\rho_{\mathrm{G}}\right)$, which describes the extent to which the same suite of genetic factors influence covariation of primary and permanent caries scores. This parameter indicates whether the same or different genetic effects are acting on the two traits. Significance of this parameter (whether $\rho_{\mathrm{G}}$ differed from zero or 1) was determined by comparing constrained and unconstrained models via the likelihood ratio test. Details regarding bivariate variance components modeling are available elsewhere [Almasy et al., 1997]. All variance components analyses were performed using the Sequential Oligogenic Linkage Analysis Routines (SOLAR) software [Almasy and Blangero, 1998]. Summary statistics and data manipulations were performed in the R statistical suite ( $\mathrm{R}$ v.2.8.1, R Foundation for Statistical Computing, Vienna, Austria).

\section{Power Considerations}

Power to detect heritability ranging from $\mathrm{h}^{2}=0.10$ to 0.35 was assessed in SOLAR via 1,000 simulations of a normally-distributed, heritable trait. Overall, power calculations indicated that we had sufficient power to detect heritability in our sample. Specifically, the power estimated to detect heritability of $\mathrm{h}^{2}=0.25$ was $>99 \%$ for permanent caries scores, $81 \%$ for primary caries scores, and $>99 \%$ for total dentition caries scores. Power was near $100 \%$ to detect heritability of $\mathrm{h}^{2}>0.30$ for permanent, primary and total dentition caries scores.

\section{Results}

Dental characteristics of the sample, including agespecific caries prevalence and caries scores for both permanent and primary dentitions are shown in table 1. Caries prevalence and caries scores were similar between males and females aged 0-6 years, $14-18$ years, and $>18$ years. In contrast, primary dentition caries scores ( $\mathrm{d} 1 \mathrm{ft}$ and $\mathrm{d} 2 \mathrm{ft}$ ), but not permanent dentition caries scores (D1MFT and D2MFT), were significantly greater in males than females in the mixed dentition cohort (those individuals with at least 1 primary and at least 1 permanent tooth, aged 6-14 years). Histograms showing the distributions of primary and permanent caries scores are shown in figure 1 .

The heritability (the proportion of trait variation due to genetics after adjusting for age and sex) of caries scores for primary, permanent, and total dentition are shown in table 2. In general, dental caries were highly heritable for the primary dentition, with the cumulative effect of genes accounting for $54-70 \%$ of variation in primary caries scores. The heritability of caries scores in the permanent dentition was also moderate to high $\left(\mathrm{h}^{2}=35-55 \%\right)$, although somewhat lower than the analogous primary dentition caries scores. Overall, heritability of D1MFT/ d1ft scores (which include white-spot lesions) was slightly greater than D2MFT/d2ft caries scores (which exclude white-spot lesions). Likewise, the heritability of the proportion-DMFT/proportion-dft scores (proportion of caries teeth to at-risk teeth) was greater than the heritability of count DMFT/dft scores. 
Table 1. Observed caries prevalence and distribution across age and sex

\begin{tabular}{|c|c|c|c|}
\hline Characteristics & $\begin{array}{l}\text { Total } \\
\text { sample }\end{array}$ & Male & Female \\
\hline \multicolumn{4}{|l|}{ Age group: $0-6$ years } \\
\hline Sample size, $\mathrm{n}$ & 580 & 292 & 288 \\
\hline Caries prevalence $(\mathrm{d} 1)$ & 0.32 & 0.34 & 0.29 \\
\hline Caries prevalence (d2) & 0.29 & 0.31 & 0.27 \\
\hline $\mathrm{d} 1 \mathrm{ft}$ & $1.55 \pm 3.13$ & $1.69 \pm 3.34$ & $1.41 \pm 2.91$ \\
\hline $\mathrm{d} 2 \mathrm{ft}$ & $1.27 \pm 2.59$ & $1.40 \pm 2.75$ & $1.13 \pm 2.42$ \\
\hline \multicolumn{4}{|l|}{ Age group: 6-14 years } \\
\hline Sample size, $\mathrm{n}$ & 700 & 357 & 343 \\
\hline Caries prevalence $(\mathrm{D} 1+\mathrm{d} 1)^{\mathrm{a}}$ & 0.77 & 0.81 & 0.72 \\
\hline Caries prevalence $(\mathrm{D} 2+\mathrm{d} 2)^{\mathrm{b}}$ & 0.69 & 0.75 & 0.63 \\
\hline $\mathrm{d} 1 \mathrm{ft}^{\mathrm{b}} \mathrm{P}$ & $2.35 \pm 2.72$ & $2.68 \pm 2.93$ & $1.98 \pm 2.42$ \\
\hline $\mathrm{d} 2 \mathrm{ft}^{\mathrm{b}}$ & $2.15 \pm 2.61$ & $2.46 \pm 2.79$ & $1.79 \pm 2.33$ \\
\hline D1MFT & $1.88 \pm 2.83$ & $1.75 \pm 2.73$ & $2.02 \pm 2.92$ \\
\hline D2MFT & $1.13 \pm 1.85$ & $1.01 \pm 1.71$ & $1.26 \pm 1.97$ \\
\hline \multicolumn{4}{|l|}{ Age group: $14-18$ years } \\
\hline Sample size, $\mathrm{n}$ & 191 & 91 & 100 \\
\hline Caries prevalence (D1) & 0.81 & 0.82 & 0.79 \\
\hline Caries prevalence (D2) & 0.76 & 0.78 & 0.75 \\
\hline D1MFT & $5.23 \pm 5.26$ & $5.62 \pm 5.61$ & $4.87 \pm 4.92$ \\
\hline D2MFT & $3.92 \pm 3.84$ & $3.89 \pm 3.48$ & $3.95 \pm 4.15$ \\
\hline \multicolumn{4}{|l|}{ Age group: $18+$ years } \\
\hline Sample size, $\mathrm{n}$ & 1,129 & 419 & 710 \\
\hline Caries prevalence (D1) & 0.96 & 0.96 & 0.96 \\
\hline Caries prevalence (D2) & 0.96 & 0.95 & 0.96 \\
\hline D1MFT & $10.26 \pm 6.41$ & $10.09 \pm 6.44$ & $10.36 \pm 6.40$ \\
\hline D2MFT & $9.10 \pm 5.86$ & $8.95 \pm 5.92$ & $9.19 \pm 5.83$ \\
\hline
\end{tabular}

D1 = Caries including white-spot lesions; D2 = caries excluding whitespot lesions. Test of mean sex difference: ${ }^{\mathrm{a}} \mathrm{p}<0.05,{ }^{\mathrm{b}} \mathrm{p}<0.01$.

Sex and age explained little (0-10\%) of the variation in caries scores for the primary dentition. In contrast, sex and age explained a substantial portion (28-40\%) of variation in caries scores for the permanent dentition (table 2).

Heritability of total caries scores (counts/proportions of caries teeth across both primary and permanent dentitions) ranged from 29 to $40 \%$, and were substantially lower than the analogous primary and permanent caries heritabilities. To further explore this observation, we calculated the genetic correlation $\left(\rho_{\mathrm{G}}\right)$ between the D1MFT proportion among permanent and the d1ft proportion among primary teeth. $\rho_{\mathrm{G}}$ measures the extent of overlap in effects of the genes which influence the two traits. The genetic correlation between D1MFT and $\mathrm{d} 1 \mathrm{ft}$ proportions was $\rho_{\mathrm{G}}=0.43$, significantly greater than zero (non-pleiotropic; $\mathrm{p}<0.01$ ), and significantly less than one (fully pleiotropic; $p<0.01$ ). This magnitude of
$\rho_{\mathrm{G}}$ indicates that approximately $18 \%$ (the square of $\rho_{\mathrm{G}}$ ) of the cumulative effect of all genes involved is shared between primary and permanent dentition. In other words, some genes affect caries similarly in both primary and permanent dentitions, whereas the majority of genetic effects (82\%) are due to dentition-specific caries genes or differential effect sizes of genes common to both primary and permanent caries. The genetic correlation between $\mathrm{D} 2 \mathrm{MFT}$ and $\mathrm{d} 2 \mathrm{ft}$ proportions was similar (data not shown).

Because the heritability estimates differed slightly between caries scores including and excluding white-spot lesions, we investigated the heritability of each component $(\mathrm{D} / \mathrm{d}, \mathrm{M}$ or F/f) of the DMFT and dft scores. Figure 2 shows the heritability of sound (without decay), white-spot lesion, decayed (D2/d2), missing, and filled teeth for permanent and primary dentition. As expected, the number of teeth with white-spot lesions or decay was highly heritable in both the permanent and primary dentitions. On the other hand, treated caries, such as missing and filled teeth, were less heritable, as was expected since these components are highly reflective of non-genetic factors such as access to oral health care.

\section{Discussion}

To our knowledge, this is the first comprehensive heritability study for dental caries focusing on both primary and permanent dentitions in individuals across a wide range of ages. We observed that caries scores were moderately to highly heritable, and that different genes may influence variation in primary versus permanent dentition. Our results echo previous reports indicating the important role of genes on the development of dental caries [Horowitz et al., 1958; Goodman et al., 1959; Boraas et al., 1988; Conry et al., 1993; Bretz et al., 2006]. Estimates for the heritability of caries scores from our study were generally lower than in previous reports, possibly due to differences in population, metrics for quantifying caries, and study designs (twins vs. family studies).

In general, we observed greater primary caries prevalence and greater primary caries scores in boys than girls (particularly in those aged 6-14 years), but similar permanent caries prevalence and permanent caries scores in men and women. Sex differences in caries prevalence have been reported for many years, although this remains controversial and may differ by culture and geographic region [Wyne and Khan, 1998; Khan et al., 2001; Antunes et al., 2003; Wyne, 2008]. In most reports, fe- 
Fig. 1. Histograms of distributions of dental phenotypes. Histograms showing distributions of permanent D1MFT (a), permanent proportion D1MFT (b), primary $\mathrm{d} 1 \mathrm{ft}(\mathbf{c})$ and primary proportion $\mathrm{d} 1 \mathrm{ft}(\mathbf{d})$.

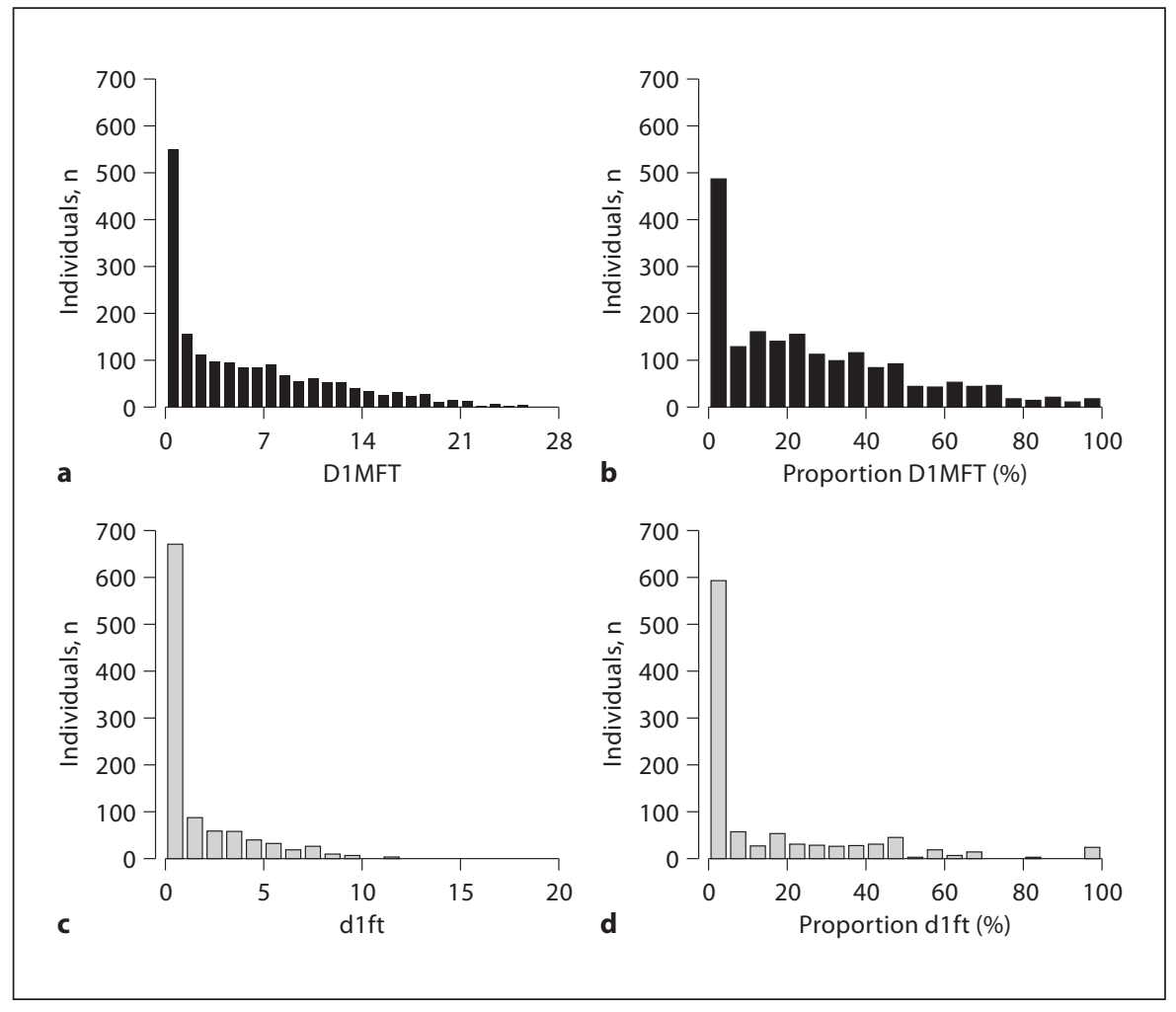

Table 2. Heritability estimates for primary, permanent, and total dentition caries scores

\begin{tabular}{llll}
\hline & $\mathrm{h}^{2}$ & $\mathrm{SE}$ & $\mathrm{R}^{2}$ \\
\hline Primary dentition (n = 1,058) & & & \\
d1ft & 0.57 & 0.10 & 0.00 \\
Proportion d1ft & 0.70 & 0.09 & 0.09 \\
d2ft & 0.54 & 0.10 & 0.00 \\
$\quad$ Proportion d2ft & 0.66 & 0.09 & 0.10 \\
Permanent dentition (n =1,949) & & & \\
$\quad$ D1MFT & 0.49 & 0.06 & 0.31 \\
$\quad$ Proportion D1MFT & 0.55 & 0.05 & 0.28 \\
D2MFT & 0.35 & 0.07 & 0.40 \\
$\quad$ Proportion D2MFT & 0.44 & 0.07 & 0.39 \\
Total dentition (n =2,445) & & & \\
$\quad$ D1MFT + d1ft & 0.34 & 0.04 & 0.33 \\
$\quad$ Proportion D1MFT + d1ft & 0.40 & 0.04 & 0.31 \\
D2MFT + d2ft & 0.29 & 0.05 & 0.38 \\
Proportion D2MFT +d2ft & 0.33 & 0.05 & 0.37 \\
\hline
\end{tabular}

$\mathrm{h}^{2}=$ Heritability (proportion of phenotype variance due to genetic factors); $\mathrm{SE}=$ standard error of $\mathrm{h}^{2} ; \mathrm{R}^{2}=$ total proportion of phenotype variance due to effects of sex and age. All $\mathrm{p}$ values for heritability estimates are $\leq 0.01$.

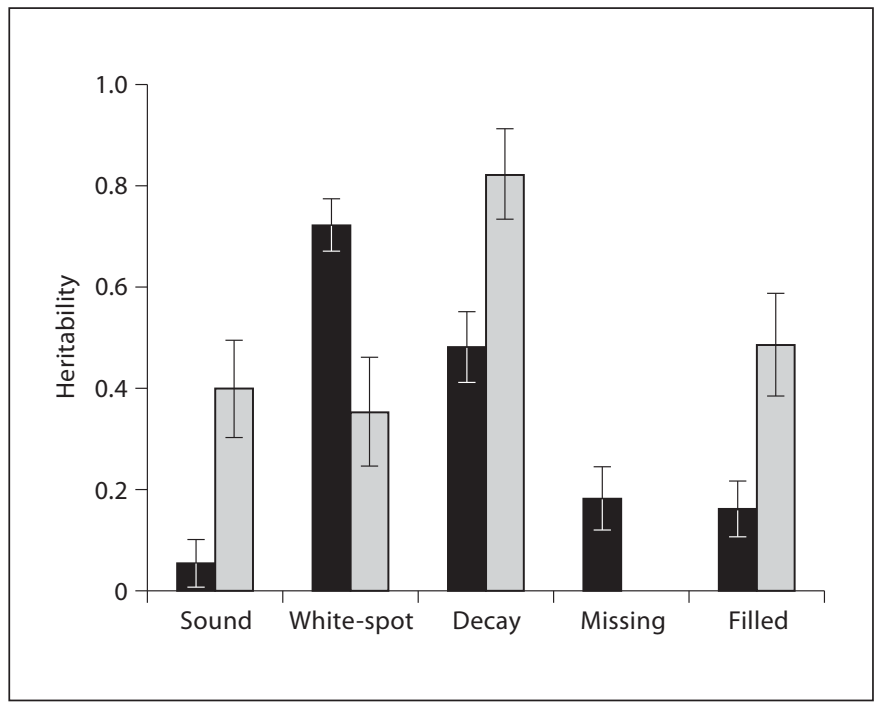

Fig. 2. Heritability of DMFT scores by components. Heritability of sound, white-spot lesion, decay, missing, and filled teeth for permanent dentition (black bars) and primary dentition (gray bars). Error bars represent $\pm \mathrm{SE}$. 
males typically exhibit higher caries rates than males across all age groups [Fujita et al., 2007], possibly due to dietary or other environmental factors, earlier teeth eruption in girls, pregnancy [Lukacs and Largaespada, 2006], or sex-dependent genetic factors [Vieira et al., 2008]. It is unclear why caries prevalence was greater in boys than girls in our study, or what factors may contribute to this difference in the COHRA study population, but these questions are under active investigation by our research group.

The high heritability of caries phenotypes [Horowitz et al., 1958; Goodman et al., 1959; Boraas et al., 1988; Conry et al., 1993; Bretz et al., 2006] has led to gene mapping efforts to find specific genetic loci contributing to caries susceptibility [Acton et al., 1999; Shuler, 2001; Chaussain-Miller et al., 2006; de Soet et al., 2006; Deeley et al., 2008; Vieira et al., 2008]. Each of these studies has identified genes involved in processes related to development of caries, including saliva composition and flow, tooth morphology, dietary and taste preferences, and enamel formation. However, most of these findings were reported in cohorts of very specific age ranges, and therefore may not be reproducible across all ages.

The reduced heritability observed in the analysis of total dentition caries led us to speculate that the set of genes affecting caries in both primary and permanent dentitions may be a subset of all caries genes. Therefore, we used bivariate modeling methods to test whether the set of genes affecting susceptibility to caries in primary teeth differed from the set of genes affecting susceptibility to caries in permanent teeth. Results of our bivariate models indicated that genetic determinants of caries in primary teeth largely differed from those in permanent teeth. This may be due to different suites of genes acting in disease genesis in primary and permanent dentitions, or due to genes that differentially exert their effects on caries development across the life course (i.e. genes that have weak effects on primary caries but large effects on permanent caries and vice versa), or both. Despite identification of some caries-related genes, the high heritability of caries phenotypes indicates that many genetic risk factors are yet to be determined.

To this end, a major goal of heritability analysis is to inform downstream gene mapping studies by identifying which 'phenotype' (the specific metric used to quantify caries) will yield the highest power to detect caries genes. In our study, we compared the heritability between two diagnostic thresholds of tooth decay by calculating caries scores including and excluding whitespot lesions (D1MFT/d1ft vs. D2MFT/d2ft) and by ana- lyzing DMFT/dft components separately. For both primary and permanent dentition, D1MFT/d1ft caries scores exhibited higher heritability than D2MFT/d2ft caries scores. Moreover, for primary and permanent dentition, both white-spot lesion and decay components exhibited high heritability in the component-specific analysis. Taken together, these results indicate that white-spot lesions contribute to (rather than detract from) the heritability of caries scores and may be regulated by the same genetic factors as more advanced stages of tooth decay. Therefore, caries phenotypes that include white-spot lesions may be more powerful for gene mapping. Likewise, proportion caries scores, which (unlike the count DMFT/dft scores) take into account both the number of affected teeth and the number of atrisk teeth, demonstrated the greatest heritability, and therefore may be the most suitable phenotypes for future genetic studies.

The rich set of phenotypes in the COHRA study, such as caries scores including and excluding white-spot lesions and, most importantly, caries scores for both primary and permanent dentition, is one of the major strengths of this study. Another strength of our family study design is that, compared to twin studies, it is less susceptible to inflated heritability estimates caused by non-genetic familial factors. This is because twins (including dizygotic twins) are likely to share unmeasured environmental and behavior exposures that track with kinship coefficient (degree of relatedness), whereas inclusion of more distant relatives, such as half-siblings raised separately, cousins, and parent-offspring pairs (reared at different times and in different environments), help insulate heritability estimates from the effects of familial non-genetic exposures. Our study also benefits from an overall sample size that is substantially larger than previous heritability studies [Horowitz et al., 1958; Goodman et al., 1959; Boraas et al., 1988; Conry et al., 1993; Bretz et al., 2006], and has high power to detect heritability. Further, family relationships were confirmed by genotype results from 72 ancestry-informative SNP markers. Finally, we accounted for potential study weaknesses arising from phenotype non-normality and sampling method through appropriate analyses and inference. We accounted for inflated asymptotic $p$ values that may arise due to departure from normality by calculating empirical significance (based on permutations) of heritability. We also restrict our conclusions to the underlying sampling population derived from a demographic with poorer oral health and less access to oral health care than much of the US. The applicability of our 
findings to other US demographic strata is unknown at this time.

In conclusion, we have demonstrated the heritability of dental caries traits and provided evidence that different genes, or genes with differential effect sizes, are involved in tooth decay in primary and permanent dentitions. Moreover, we have shown that, from a genetic perspective, white-spot lesions are an important component of tooth decay, and we surmise that future studies of white-spot lesions along with more advanced tooth decay may lead to better understanding of the genetic determinants and biological pathways leading to dental caries.

\section{Acknowledgments}

We thank the families for their participation in the study and contributing to the enhancement of oral health information in these Appalachian rural communities. We also thank the field staff of the data collection and coordination sites, as well as the participating community health care organizations. In Pennsylvania: University of Pittsburgh, Bradford, Center for Rural Health Practice; McKean County Dental Center, Bradford; Cornerstone
Care Community Medical and Dental Center, Burgettstown; and UPMC Braddock Hospital. In West Virginia: the Camden-onGauley Medical Center; Community Health Clinic of Nicholas County; Richwood Area Community Hospital; Summersville Memorial Hospital; and the Webster County Memorial Hospital. We appreciate the assistance of the GORGE Connection Rural Health Education Partnership Board, the Webster-Nicholas Rural Health Education Consortium Board, the West Virginia Rural Health Education Partnerships program, the Nicholas and Webster Boards of Education, the UPMC Braddock Community Advisory Board, and the numerous individuals and social service agencies that helped develop the Center for Oral Health Research in Appalachia.

This study was supported by grants from the National Institute of Dental and Craniofacial Research (NIDCR; including R01DE014899 and U01-DE018903), which is part of the National Institutes of Health (NIH) Genes and Environment Initiative (GENEVA). CIDR is fully funded through a federal contract from the NIH to The Johns Hopkins University, contract number HHSN268200782096C. Funds for genotyping were provided by the NIDCR through CIDR's NIH contract. Additional support was provided by the University of Pittsburgh School of Dental Medicine, the West Virginia University School of Dentistry and Eberly College of Arts and Sciences. The content is solely the responsibility of the authors and does not necessarily represent the official views of the NIDCR or the NIH.

\section{References}

Acton RT, Dasanayake AP, Harrison RA, Li Y, Roseman JM, Go RC, Wiener H, Caufield PW: Associations of MHC genes with levels of caries-inducing organisms and caries severity in African-American women. Hum Immunol 1999;60:984-989.

-Almasy L, Blangero J: Multipoint quantitativetrait linkage analysis in general pedigrees. Am J Hum Genet 1998;62:1198-1211.

-Almasy L, Dyer TD, Blangero J: Bivariate quantitative trait linkage analysis: pleiotropy versus co-incident linkages. Genet Epidemiol 1997; 14:953-958.

-Anderson M: Risk assessment and epidemiology of dental caries: review of the literature. Pediatr Dent 2002;24:377-385.

-Antunes JL, Junqueira SR, Frazao P, Bispo CM, Pegoretti T, Narvai PC: City-level gender differentials in the prevalence of dental caries and restorative dental treatment. Health Place 2003;9:231-239.

Boraas JC, Messer LB, Till MJ: A genetic contribution to dental caries, occlusion, and morphology as demonstrated by twins reared apart. J Dent Res 1988;67:1150-1155.

- Bretz WA, Corby PM, Hart TC, Costa S, Coelho MQ, Weyant RJ, Robinson M, Schork NJ: Dental caries and microbial acid production in twins. Caries Res 2005a;39:168-172.
Bretz WA, Corby PM, Melo MR, Coelho MQ, Costa SM, Robinson M, Schork NJ, Drewnowski A, Hart TC: Heritability estimates for dental caries and sucrose sweetness preference. Arch Oral Biol 2006;51: 1156-1160.

Bretz WA, Corby PM, Schork NJ, Robinson MT, Coelho M, Costa S, Melo Filho MR, Weyant RJ, Hart TC: Longitudinal analysis of heritability for dental caries traits. J Dent Res 2005b;84:1047-1051.

-Centers for Disease Control and Prevention: Total tooth loss among persons aged $\geq 65$ years: selected states, 1995-1997. JAMA 1999;281: 1264-1266.

-Centers for Disease Control and Prevention: Public health and aging: retention of natural teeth among older adults: United States, 2002. MMWR Morb Mortal Wkly Rep 2003 52:1226-1229.

-Chaussain-Miller C, Fioretti F, Goldberg M, Menashi S: The role of matrix metalloproteinases (MMPS) in human caries. J Dent Res 2006; 85:22-32.

-Conry JP, Messer LB, Boraas JC, Aeppli DP, Bouchard TJ Jr: Dental caries and treatment characteristics in human twins reared apart. Arch Oral Biol 1993;38:937-943.
Deeley K, Letra A, Rose EK, Brandon CA, Resick JM, Marazita ML, Vieira AR: Possible association of amelogenin to high caries experience in a Guatemalan-Mayan population. Caries Res 2008;42:8-13

de Soet J, Driel L, Pleijster J, Morre S, GemertSchriks M: CD14 gene polymorphism in relation to caries. Caries Res 2006;40:349.

Fujita H, Asakura K, Ogura M: Age- and sex-related dental caries prevalence in Japanese from the Jōmon period. J Oral Biosci 2007; 49:198-204.

Goodman HO, Luke JE, Rosen S, Hackel E: Heritability in dental caries, certain oral microflora and salivary components. Am J Hum Genet 1959;11:263-273.

Grembowski D, Conrad DA, Milgrom P: Dental care demand among children with dental insurance. Health Serv Res 1987;21:755-775.

Horowitz SL, Osborne RH, Degeorge FV: Caries experience in twins. Science 1958;128:300301.

Hunter PB: Risk factors in dental caries. Int Dent J 1988;38:211-217.

Huttlinger KW, Purnell LD: People of Appalachian heritage; in Purnell LD, Paulanka BJ (eds): Transcultural Health Care: A Culturally Competent Approach. Philadelphia, FA Davis, 1998, pp 95-112. 
Janes GR, Blackman DK, Bolen JC, Kamimoto LA, Rhodes L, Caplan LS, Nadel MR, Tomar SL, Lando JF, Greby SM, Singleton JA, Strikas RA, Wooten KG: Surveillance for use of preventive health-care services by older adults, 1995-1997. MMWR CDC Surveill Summ 1999;48:51-88.

Khan NB, Ghannam NA, AI Shammery AR, Wyne AH: Caries in primary school children: prevalence, severity and pattern in AlAhsa, Saudi Arabia. Saudi Dent J 2001;12: 71-74.

Liu H, Deng H, Cao CF, Ono H: Genetic analysis of dental traits in 82 pairs of female-female twins. Chin J Dent Res 1998;1:12-16.
Lukacs JR, Largaespada LL: Explaining sex differences in dental caries prevalence: saliva, hormones, and 'life-history' etiologies. Am J Hum Biol 2006;18:540-555.

Marosy B, Romm J, Hetrick K, Doheny K, Pugh E, Tsai Y: Development of a low cost SNP barcode panel (abstract 2653f). Am Soc Hum Genet 57th Ann Meet, San Diego, 2007.

O'Connell JR, Weeks DE: Pedcheck: A program for identification of genotype incompatibilities in linkage analysis. Am J Hum Genet 1998;63:259-266.

Polk DE, Weyant RJ, Crout RJ, McNeil DW, Tarter RE, Thomas JG, Marazita ML: Study protocol of the center for oral health research in Appalachia (COHRA) etiology study. BMC Oral Health 2008;8:18.
-Shuler CF: Inherited risks for susceptibility to dental caries. J Dent Educ 2001;65:10381045.

Vieira AR, Marazita ML, Goldstein-McHenry T: Genome-wide scan finds suggestive caries loci. J Dent Res 2008;87:435-439.

Wyne AH: Caries prevalence, severity and pattern in preschool children. J Contemp Dent Pract 2008;9:24-31.

Wyne AH, Khan NB: Caries prevalence in 2 and 3 year old children of Adelaide, Australia. Odontostomatol Trop 1998;21:22-23; erratum in Odontostomatol Trop 1999;22:24. 